\title{
Automated Analysis of Large Datasets Acquired with STEM Diffraction Mapping
}

\author{
Christoph Gammer ${ }^{1,2}$. \\ ${ }^{1 .}$ National Center for Electron Microscopy, Molecular Foundry, Lawrence Berkeley National \\ Laboratory, Berkeley, CA, USA. \\ 2. Physics of Nanostructured Materials, Faculty of Physics, University of Vienna, Austria.
}

STEM diffraction mapping yields a very rich dataset that can be analyzed in multiple ways after the experiment. The present work presents recent advances in software development that enable fast and easy processing of large datasets.

In scanning nanobeam electron diffraction a sample region is scanned by a converged electron probe and for each probe position an electron diffraction pattern is recorded along with the regular ADF-signal. Thus, each pixel of the resulting STEM map is a full diffraction pattern for the corresponding beam position. Acquisition of a map by recording a diffraction pattern and shifting the electron probe is limited to a small number of probe positions, due to the time needed to record a diffraction pattern. Therefore, the electron detector is used in continuous recording mode and synced with the scan coils. This technique is only limited by the speed of the CCD camera, yielding up to $20 \mathrm{f} / \mathrm{s}$. The acquisition is fully automated and large maps can be recorded in around 30 minutes. Recent developments in fast electron detectors enable recording datasets in a significantly shorter time. A Gatan K2 IS direct electron detector operating at a frame rate of $400 \mathrm{f} / \mathrm{s}$ was used to record diffraction maps with $256 \times 256$ probe positions in less than 3 minutes. The resulting dataset consists of more than 65,500 diffraction patterns, with around 2000x2000pixels each [1].

To evaluate the diffraction map, custom DigitalMicrograph scripts were written. Figure 1a shows a screenshot of the plugin during reconstruction of a dark-field image. The dark-field image is reconstructed by calculating the mean intensity in the virtual aperture at each probe position $[1,2]$. Using a graphical user interface, arbitrary virtual apertures can be created. Automated routines allow to create virtual apertures specifically designed according to the positions of the diffracted peaks. To reconstruct a dark-field image from a large dataset, the data has to be binned prior to loading to memory or stored in individual files, which are then processed sequentially. To overcome this limitation, the HDF5 format can be used, allowing to address the necessary data directly without loading the entire series into memory. These advances allow live dark-field reconstruction of the acquired datasets, enabling a more detailed analysis of the recorded sample area.

The diffraction patterns acquired by STEM diffraction mapping contain rich structural information on the sample. Utilizing the locations of the diffracted peaks allows generating strain maps. The resulting maps combine nanometer resolution with a high precision and a very large field of view [3]. Figure 1b shows a screenshot of the plugin during calculation of a strain map. Template matching is used to detect the diffracted peaks with sub-pixel accuracy. The graphical user interface allows to create the template and set-up optimized pre-filter parameters prior to the automated analysis. Optimized computation routines are used allowing to process strain maps with up to $512 \times 512$ probe positions in a relatively short time.

Working with the entire dataset rather than with an individual image, does not only allow for a detailed 
analysis of the structure but enables greater quantification of the microstructure. Owing to improved acquisition and computational routines it is now possible to generate a single large dataset containing all the structural information for the scanned sample area at the given orientation. The dataset can be analyzed in multiple ways after the experiment to generate more quantitative and informative images than possible with conventional imaging techniques.

[1] C. Gammer, V.B. Ozdol, C.H. Liebscher and A.M. Minor. Ultramicroscopy 155 (2015) p.1.

[2] Z. Zeng, X. Zhang, K. Bustillo, K. Niu, C. Gammer, J. Xu and H. Zheng. Nano Letters 15 (2015) p.5214.

[3] V.B. Ozdol, C. Gammer, X.G. Jin, P. Ercius, C. Ophus, J. Ciston and A.M. Minor. Applied Physics Letters 106 (2015) 253107.

[4] The authors acknowledge support by the Austrian Science Fund (FWF):[J3397] and the Molecular Foundry, Lawrence Berkeley National Laboratory, which is supported by the U.S. Dept. of Energy under Contract \# DE-AC02-05CH11231.
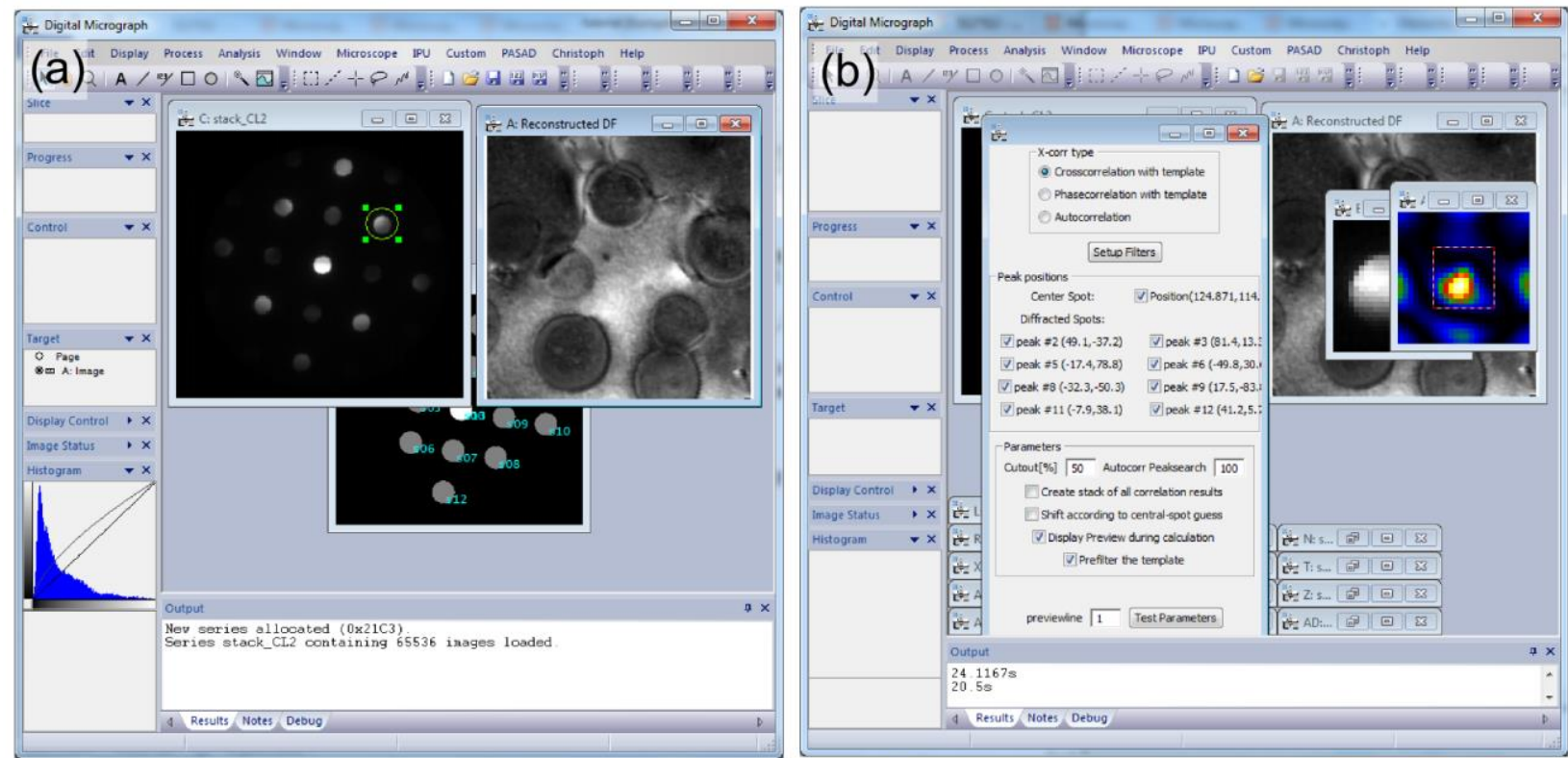

Figure 1. Screenshots of a custom DigitalMicrograph plugin allowing to evaluate diffraction maps. (a) Arbitrary virtual apertures can be drawn in the diffraction pattern and are used to generate reconstructed dark-field images. (b) Strain-mapping is carried out by detecting the shift of selected peaks using template-matching. 